\title{
Emil Julius Gumbel
}

\section{Innovativer Statistiker und engagierter Pazifist}

\author{
Ulrich Rendtel (D) - Ulrike C. Wasmuht • Peter-Theodor Wilrich
}

Eingegangen: 9. April 2021 / Angenommen: 7. September 2021 / Online publiziert: 19. November 2021 (C) Der/die Autor(en) 2021

Zusammenfassung Emil J. Gumbel ist der Namensgeber der jährlichen GumbelVorlesung auf der Statistischen Woche. Leider ist der Namensgeber dieser Vorlesung nur noch wenigen Teilnehmern und Vortragenden bekannt. Dieser Artikel möchten diesem Defizit abhelfen. Denn Gumbel war nicht nur der Namensgeber irgendeiner statistischen Verteilung.

Der Aufsatz beschreibt den Lebensweg Gumbels vom Weltkriegsteilnehmer zu einem weithin bekannten Pazifisten, der die politischen Morde in der frühen Weimarer Republik mit statistischen Schlußweisen analysierte. Seine Zahlenwerke legten die Defizite der Rechtssprechung in der Weimarer Republik offen. Die Richter kompromittierten sich mit ihren Urteilen. Seine Statistik-Professur an der Universität Heidelberg verlor er nach Angriffen der NS-Studenten und einer deutsch-nationalistischen Universitätsleitung noch vor Beginn der NS-Herrschaft. Wir schildern Gumbels Emigration erst nach Frankreich und von da in die USA und seine vergeblichen Versuche, wieder an deutschen Universitäten aufgenommen zu werden.

Im zweiten Teil skizzieren wir Gumbels wissenschaftliches Werk im Bereich der Statistik der Extremwerte. Gumbel schrieb nicht nur den Klassiker dieses Statistik-Bereichs. Er baute auch vielen Ingenieuren einen fachlichen Zugang mit leicht berechenbaren und gut interpretierbaren Diagrammen.

Schließlich schildert einer der Autoren, der Gumbel noch persönlich kannte, seine Erinnerungen an den freundlichen und interessierten Gastprofessor aus den USA.

Schlüsselwörter Pazifismus · Statistik der Gerichtsurteile · „Gumbel-Krawalle“ . Extremwerte $\cdot$ Gumbel-Verteilung

Ulrich Rendtel $(\varangle) \cdot$ Ulrike C. Wasmuht $\cdot$ Peter-Theodor Wilrich

Freie Universitat Berlin, Berlin, Deutschland

E-Mail: ulrich.rendtel@fu-berlin.de 


\section{Emil Julius Gumbel}

An innovative statistician and a dedicated pacifist

Abstract The annual Gumbel lecture of the Statistische Woche is dedicated to Emil J. Gumbel. Unfortunately, Emil J. Gumbel is known to only a few of the participants and presenters of this lecture. This article intends to remedy this deficit. Because Gumbel was not only a person whereby some statistical distribution was named after.

The article describes Gumbel's path of life from a world war I participant to a widely known pacifist, who analyzed the political murders during the early phase of the German Weimar Republic by statistical means. His computations revealed the deficits of jurisdiction during the Weimar Republic. The judges compromised themselves by their verdicts. Gumbel lost his position of a professor of statistics at the University of Heidelberg after attacks of Nazi students and a German-nationalist university management before the Nazis took over the political power. We describe the emigration of Gumbel first to France and from there to the US and his unsuccessful attempts to reinstall his former position at a German University.

In the second part we outline Gumbel's scientific work in the area of the statistics of extremes. Gumbel did not only write a classical monograph on this statistical field, but for many engineers he also generated an access with easy to compute and easy to interpret diagrams. Finally, one of the authors, who did know Gumbel personally, describes his memories of a friendly and interested guest professor from the US.

Keywords Pacifism - Statistics of sentences - Gumbel clashes · Extrem values · Gumbel Distribution

\section{Einleitung}

Seit 2006 findet anläßlich der Statistischen Woche jährlich eine Gumbel-Vorlesung statt. Diese Plenarveranstaltung wurde vom Vorstand der Deutschen Statistischen Gesellschaft unter Karl Mosler eingeführt. Im Vorstand suchte man nach einem passenden Namesgeber. Die Wahl fiel schließlich auf Emil J. Gumbel, einen deutschen Statistiker, nach dem eine sogar Verteilung benannt war. Abbildung 1 zeigt ein Protaitfoto von Emil J. Gumbel.

Diese Plenarveranstaltung ist eher methodisch orientiert im Gegensatz zu der 2011 etablierten Heinz-Grohmann-Vorlesung, die stark anwendungsorientiert ist. Doch während der Namensgeber der Heinz-Grohmann-Vorlesung und seine Rolle für die Statistik in dieser Zeitschrift ausführlich vorgestellt wurden (Chlumsky 2012), ist über den Namensgeber der Gumbel-Vorlesung bisher in dieser Zeitschrift nur wenig geschrieben worden. Auch auf der Website der Deutschen Statistischen Gesellschaft findet man im Abschnitt Gumbel-Vorlesung nur einige Meilensteine aus Gumbels Leben. Einige Kollegen, die sich mit technischer Statistik befassen, werden vielleicht eine „Gumbel-Verteilung“ kennen, die im Bereich der Extremwertverteilungen eine wichtige Rolle spielt. Dass der Namensgeber ein Deutscher war, ist noch weniger 
Abb. 1 Emil Julius Gumbel (1891-1966)

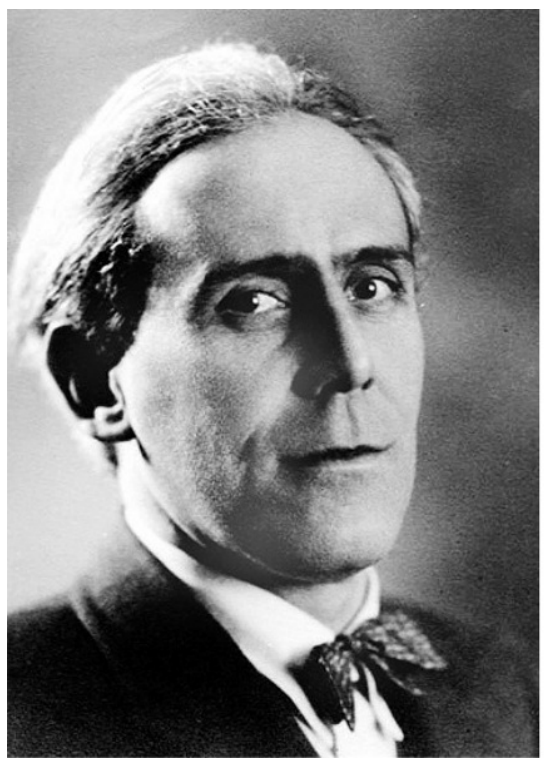

bekannt. Und leider nehmen auch die Gumbel-Lecturer auf den Namensgeber keinen Bezug, wie das dagegen bei der Heinz-Grohmann-Vorlesung üblich ist.

Damit geschieht Emil Julius Gumbel allerdings Unrecht. Gumbel ist in der Bundesrepublik eher als politischer Autor der Weimarer Republik bekannt und - anders als im anglo-amerikanischen Raum - weniger als bedeutender Statistiker (vgl. die Enzyklopädien Johnson und Kotz 1997 und Heyde und Seneta 2001 sowie Salsburg 2001). Der politische Akteur der Weimarer Republik war damals allerdings als Intellektueller in der Öffentlichkeit sehr bekannt. Sein wissenschaftliches Werk und vor allen Dingen dessen praktische Anwendung fanden bei Ingenieuren weltweit Anwendung. Es lohnt sich durchaus, sich mit der wechselvollen Lebensgeschichte dieses innovativen Statistikers und engagierten Pazifisten zu befassen (siehe auch Fernández et al. 2019, Holl 1988, Vogt 1991). Deshalb sollen in diesem Beitrag Person und Werk des Namensgebers der Gumbel-Vorlesung besonders gewürdigt und einer breiteren Öffentlichkeit innerhalb der statistischen Community erschlossen ${ }^{1}$ werden.

\footnotetext{
${ }^{1}$ Im Bereich der Geschichtswissenschaft wurde Gumbel anlässlich seines 100. Geburtstags öffentlich gewürdigt. Aus Anlaß dieses Jubiläums erschienen zwei Biographien der Historiker und Mathematiker Anette Vogt (1991) und Christian Jansen (1991). 2012 wurde aus aktuellem Anlaß der NSU-Morde auf Gumbels Auseinandersetzung mit dem rechten Terror verwiesen, siehe auch „Rechnen gegen den Terror“, Furth (2012) sowie Lahusen (2012); 2019 wurde von einer interdisziplinären Forschungsgruppe (Lexuri Fernández, Matthias Scherer (beide TU München) sowie Werner Frese, Annette Vogt (MPI WG Berlin) und Isabella Wiegand (TU München)) eine Wanderausstellung unter dem Titel „Emil J. Gumbel (1891-1966): Statistiker, Pazifist, Publizist. Im Kampf gegen Extreme und für die Weimarer Republik“ konzipiert. Presseberichte finden sich u.a. in FAZ, 7.8.2019, S. N3. Weiterhin erschien 2019 ein Dokumentarfilm (von David Ruf) im SWR (21.11.2019) über Gumbel unter dem Titel „Statistik des Verbrechens - Ein Mathematiker gegen die Nazis“.
} 
Die drei Autoren dieses Beitrags beleuchten unterschiedliche Aspekte von Gumbels Leben: Ulrich Rendtel und Ulrike C. Wasmuht schildern den Lebensweg Gumbels vom Pazifisten zum Professor für Statistik über die sogenannten „GumbelKrawalle"2 bis hin zu seiner Entlassung aus der Heidelberger Universität wegen „Unwürdigkeit“" noch zu Zeiten der Weimarer Republik und zu der unversöhnlichen und unrühmlichen Haltung der Heidelberger Universität im Nachkriegsdeutschland. Dabei beleuchten sie insbesondere die von Gumbel genutzte alte Tradition einer Argumentation mit statistischen Mitteln in kontroversen politischen Auseinandersetzungen, um Aufmerksamkeit für eine Sache zu erreichen, investigativ Missstände aufzudecken oder sogar Veränderungen bei den Obrigkeiten durchzusetzen. Peter-Theodor Wilrich dagegen hat Gumbel noch persönlich kennengelernt. Wilrichs Hauptaugenmerk richtet sich auf die statistischen Eigenschaften der Gumbel-Verteilung sowie deren praktische Relevanz. Und er berichtet über Gumbel als einen freundlichen und neugierigen Gastwissenschaftler aus den USA.

\section{Gumbel der Pazifist}

Emil Julius Gumbel wurde 1891 in München als Sohn wohlhabender, bürgerlich liberaler, jüdischer Eltern geboren. Gumbel studierte in München Mathematik und Nationalökonomie an der Ludwig-Maximilians-Universität. 1913 erhielt er das Diplom als Versicherungsmathematiker und begann als Wissenschaftlicher Assistent im Seminar für Statistik und Versicherungswesen bei Professor Georg von Mayr, dem Gründer des „Allgemeinen Statistischen Archiv“ (1890) und dem ersten Vorsitzenden der Deutschen Statistischen Gesellschaft. Schon ein Jahr später schloss er seine Promotion über die Interpolation von Bevölkerungszeitreihen unmittelbar vor Beginn des Ersten Weltkriegs ab. Gumbel meldete sich - der allgemeinen Kriegsbegeisterung folgend - als Freiwilliger zum Kriegsdienst.

Es ist nicht belegt, dass Gumbel selbst im Fronteinsatz war. Doch sein Bruder und der Sohn seines auf ihn einflussreichen Onkels starben schon in den ersten Kriegsmonaten. Angesichts dieser Geschehnisse trat Gumbel bereits 1915 dem pazifistischen „Bund neues Vaterland“, der späteren „Liga für Menschenrechte“, bei. Der Liga gehörten unter anderem Ernst Reuter sowie Albert Einstein an. In dieser Zeit war Gumbel wegen einer Erkrankung für ein Jahr als kriegsuntauglich eingestuft worden und zog nach Berlin um, wo er an der Friedrich-Wilhelm-Universität zu Berlin, der heutigen Humboldt-Universität, Vorlesungen über Physik hörte. 1916 arbeitete als Flugzeugtechniker in Berlin-Adlershof. Im Anschluss daran wechselte er zur Firma Telefunken. Später, 1921, wurde er Lehrer an der Schule für Betriebsräte des Allgemeinen Deutschen Gewerkschaftsbundes in Berlin. Während dieser Berliner Jahre engagierte er sich auch parteipolitisch und trat wegen der Auseinandersetzungen über die Kriegsanleihen in die USPD ein, weil er gegen die Zustimmung der SPD für die Kriegskredite war. Er übersetzte darüber hinaus Schriften des Pazifisten Bert-

\footnotetext{
2 Diese irreführende Bezeichnung wurde von der lokalen Presse für die von NS-Studenten verursachten Tumulte bei Veranstaltungen von Gumbel verwendet. Der Krawall wurde von NS-Studenten und nicht, wie man meinen könnte, von Gumbel initiiert.
} 
rand Russell vom Englischen ins Deutsche und wurde Autor für die „Weltbühne“, die ebenfalls von einem Pazifisten, Carl von Ossietzky, geleitet wurde. Wegen seines öffentlichen Engagements gegen den Krieg war er bei den Freikorps - rechtsnationalistischen, der Weimarer Republik gegenüber feindlich eingestellten bewaffneten Gruppen - verhasst. So entging er im März 1919 nur per Zufall seiner Ermordung, als Mitglieder des Freikorps, das bereits im Januar zuvor Rosa Luxemburg und Karl Liebknecht ermordet hatte, in seine Wohnung eindrangen. Gumbel war zu diesem Zeitpunkt jedoch zu einer Konferenz des Völkerbundes, der Vorläuferorganisation der heutigen UN, nach Bern abgereist, sodass die Freikorps-Leute nur seine Wohnung verwüsten konnten. Allerdings wurde er 1920 auf einer Versammlung der Deutschen Friedensgesellschaft direkt attackiert und blutig geschlagen.

Bereits 1919 veröffentlichte Gumbel seine erste politische Schrift „Vier Jahre Lüge“ (Gumbel 1919): Das ist eine unsystematische Sammlung von Zitaten und Auszügen von Reden des Kaisers, Auszügen von amtlichen Verlautbarungen sowie Reden von Politikern und hochrangigen Militärs in der Zeit vor und während des Ersten Weltkrieges. Mit dieser kleinen Schrift wollte Gumbel folgendes erreichen: „Sie sollen einen kleinen Beitrag liefern zur Aufklärung über das unerhörte Maß der Lügen, die das kaiserliche Deutschland aufgehäuft hat, um das deutsche Volk in diesen Krieg hineinzuhetzen und es zum Durchhalten für die falsche Sache zu ermahnen. Das Tatsachenmaterial ist so groß, dass ich nicht sicher bin, das wichtigste hier gebracht zu haben. Die Zufälligkeit der Auswahl möge jeden Leser ermuntern, selbst nachzuforschen: bei welchen Gelegenheiten hast auch du dich betrügen lassen?“ (Gumbel 1919, S. 3, zitiert in: Benz 1983, S. 162) Hier zeigt sich die ,reformerische Tradition“, in der Gumbel bezüglich der Anwendung der Methoden der empirischen Sozialforschung stand: Er sammelte zunächst qualitativ Daten aus veröffentlichtem Material, das er zusammenstellte, um bei seinen Lesern und Leserinnen eine unmissverständliche Botschaft zu übermitteln. Die Schlussfolgerungen aus diesem Material überließ er seinen Lesern. Bereits im frühen 19. Jahrhundert schilderten die ersten „Enthüllungsjournalisten“ mit den Methoden der teilnehmenden Beobachtung Armutsverhältnisse und deren Begleiterscheinungen einem in besser gestellten Verhältnissen lebenden Publikum. Allen voran schrieb der englische Journalist Henry Mayhew gesellschaftskritische Berichte über Armutsverhältnisse von Einzelfällen und über für ihn ,typische Fälle“ in London (siehe dazu: Schnell et al. 2008, S. 21). Als dieser Journalist von den Obrigkeiten wegen seiner ,nichtssagenden Einzelfallschilderungen“ massiv kritisiert wurde, fing Mayhew an, seinen Berichten Statistiken beizufügen. Erst dann wurden seine Berichte von seinen Kritikern ernstgenommen. Gumbel hat aus seinen Schilderungen ohne Zahlenbelege gelernt: Seinen Arbeiten ,Zwei Jahre Mord“ (Gumbel 1921) und „Vier Jahre politischer Mord“ (Gumbel 1922) fügte er akribisch erarbeitete Tabellen bei.

\footnotetext{
3 Diese Schilderungen anhand von Presseberichten sahen zum Beispiel so aus: „Der Tagelöhner Theodor Kirchner aus der Winterstr. 4 wurde am 3. Mai ohne jedes gerichtliche Verfahren in der Kirbacherstr. 11 erschossen, obwohl er sich weder an den Kämpfen beteiligt, noch sonst strafrechtlich verfehlt hatte. Ein Gewehr hatte er vorher schon freiwillig ohne Aufforderung eingeliefert. Er hinterließ eine Witwe und 2 Kinder im Alter von 2 und 4 Jahren." (Gumbel 1922, S. 39).
} 


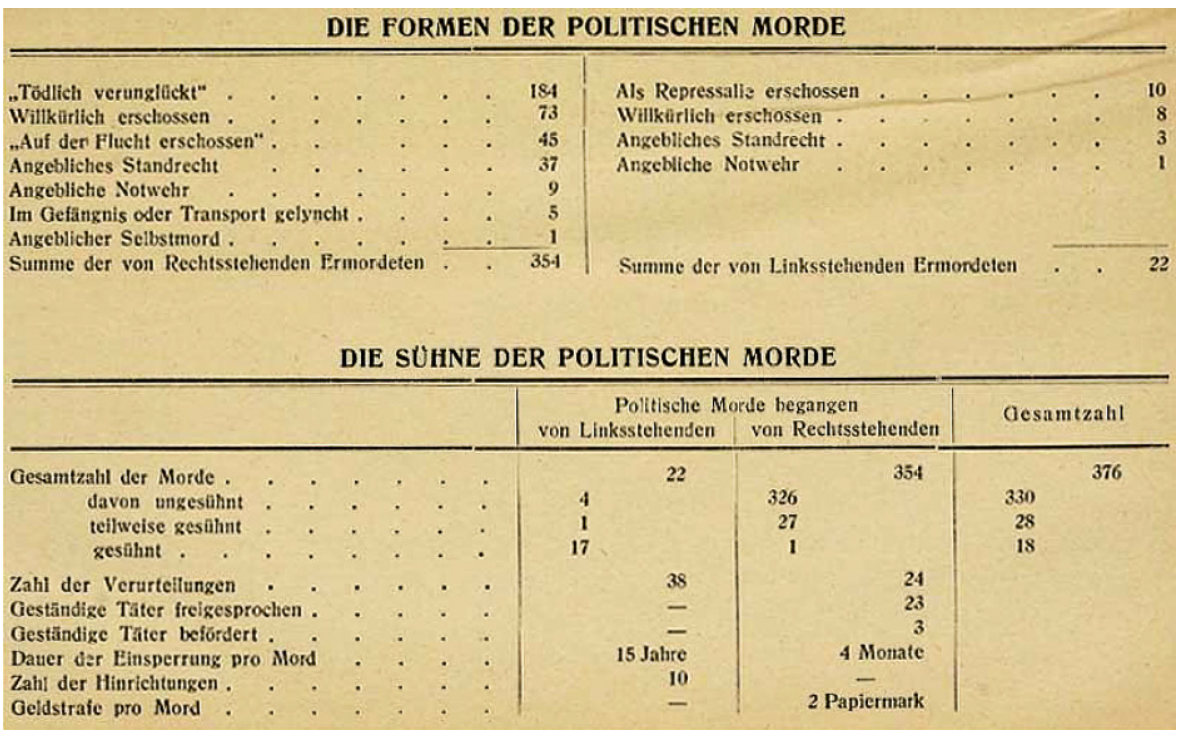

Abb. 2 Tabelle aus ,Vier Jahre Politischer Mord“

Diese Tabellen erhärteten seine Argumente, die er mit genauen Schilderungen der ihm bekannt gewordenen Ermordungen belegte. ${ }^{3}$

In den unmittelbaren Nachkriegsjahren grassierte der politische Terror gegen Vertreter der Weimarer Republik: 1921 wurde der Reichsfinanzminister Matthias Erzberger ermordet, 1922 Außenminister Walter Rathenau. Gumbel verfolgte die gerichtliche Aufarbeitung dieser Morde mit einer statistischen Methodik. In einer Zeit flammender Reden und des politischen Pathos war diese nüchterne und akribische Herangehensweise geradezu revolutionär. 1922 publizierte Gumbel unter dem Titel „Vier Jahre politischer Mord“ die juristische Aufarbeitung jener Jahre. 354 Morden von rechts standen 22 Morde von links gegenüber. Abb. 2 zeigt Gumbels statistische Auswertung dieser Morde: Auf der politisch rechten Seite endeten 326 Morde ohne Bestrafung, auf der politisch linken Seite lediglich 4. Pro Mord wurden auf der rechten Seite durchschnittlich 4 Monate Haft verhängt, während es auf der linken Seite 15 Jahre Haft oder sogar die Todesstrafe waren. Diese Publikation blieb zu Gumbels eigener Enttäuschung folgenlos. Er hatte erwartet, dass man ihn entweder wegen falscher Zahlen zur Rechenschaft ziehen würde oder aber - falls dies nicht passierte - hoffte er, dass die Verfahren wieder aufgenommen wurden und die rechten Täter höher bestraft würden. Doch nichts dergleichen geschah. Zur Verurteilung wegen Landesverrat reichte das Material nicht aus, handelte es sich doch ausschließlich um in Zeitungen publiziertes Material. Ein öffentlicher Eklat hätte Verhältnisse offenbart, die jedoch vertuscht werden sollten und eine Diskussion darüber, ob Gumbels Publikationen „Fake News“ waren, vermied man: Die Behauptungen waren beweisbar und das wusste man. Genau diese für Gumbel enttäuschende Situation zeigt gerade die Brisanz seiner Veröffentlichungen auf. Er hatte durch seine akribische Recherche und statistische Aufarbeitung einen empfindlichen Nerv der damaligen Gesellschaft getroffen. Diese Veröffentlichungen hatten eine für 
Gumbel spürbare und nachhaltige Wirkung: Er zog sich den unversöhnlichen Hass der nationalistischen Rechten und der deutschen Richterschaft zu, der sein weiteres Leben entscheidend beeinflussen sollte.

1923 erhielt Gumbel die Lehrbefähigung für Statistik an der Universität Heidelberg. Er habilitierte sich mit einer Arbeit über die ,Theorie der Statistischen Verteilungsfunktion“. Ein Jahr später - 10 Jahre nach Beginn des Ersten Weltkriegs - erklärte Gumbel anlässlich einer Schweigeminute für die Toten dieses Krieges, die ,ich will nicht sagen auf dem Feld der Unehre gefallen sind, aber doch auf grässliche Weise ums Leben kamen." Dieser Ausspruch wurde von nationalistischen Seiten zum Anlass genommen, vehement die Entfernung Gumbels von der Universität zu fordern. Die Proteste gingen dabei zunehmend von den studentischen Burschenschaften, den Korpsstudenten und dem Nationalsozialistischen Deutschen Studentenbund aus. Auch der Rektor der Universität und die Philosophische Fakultät, der Gumbel angehörte, schlossen sich der Forderung nach dessen Rauswurf an. Da der zuständige Kultusminister jedoch Gumbel verteidigte, wurde das Verfahren von der Fakultät im Mai 1925 eingestellt. Unüblich und gegen alle Gepflogenheiten verschickte die Philosophische Fakultät ihr negatives Gutachten über Gumbel an alle Universitäten in Deutschland, was einem intellektuellen Rufmord gleichkam (Jansen 1991). Die Einstellung des Verfahrens hinderte jedoch die rechtsnationalistisch eingestellte Studentenschaft nicht, Gumbel ins benachbarte Tübingen zu verfolgen, wohin er von einer Arbeitsgemeinschaft Sozialistischer Akademiker und dem örtlichen Gewerkschaftskartell zu einem Vortrag eingeladen war. Obwohl der Vortragsort kurzfristig gewechselt wurde, kam es zu einer regelrechten Saalschlacht, über die die Zeitungen als „Gumbel-Schlacht“ berichteten. Gumbel zog es vor, nach diesen Auseinandersetzungen einer Einladung nach Moskau zu folgen. Das MarXEngels-Institut bat ihn, im Rahmen einer ersten Max-Engels Gesamtausgabe die mathematischen Manuskripte von Karl Marx zum Druck vorzubereiten, siehe Vogt (1991).

Gumbels Editierung des Datenmaterials von Marx und Engels ist kein Zufall, stand er doch in derselben Tradition statistischen Arbeitens wie diese Autoren ${ }^{4}$. Auch wenn die Arbeit von Marx und Engels mit der Theorie der Politischen Ökonomie verbunden wird, so waren diese beiden Autoren harte Empiriker, die ihr Werk auf dem umfangreichen Datenmaterial aufbauten, das in jahrelanger Kleinarbeit von den im frühen 19. Jahrhundert entstandenen ,Statistical Societies“ gesammelt, aufbereitet und zur Verfügung gestellt wurde (siehe dazu auch: Desrosières 2005). Diese Gesellschaften waren Teil einer - heute würde man sagen - dezentralisierten „Graswurzelbewegung“, die vielerorts unabhängig von einander entstanden und Daten über soziale Missstände sammelten und veröffentlichten. Die Mitglieder dieser Gesellschaften sammelten nicht nur selbst Daten, sondern sie forderten auch staatliche quantitative Untersuchungen über „Arbeitsleben, Wohnsituation und Gesundheitsprobleme der Arbeiterschaft“" sowie über deren „Literaturkonsum/Lesestoff, religiöse(n) Glauben, Lebenseinstellung und die Ausstattung der Wohnung mit sanitären

\footnotetext{
4 Allerdings hatten die zu editierenden Mathematischen Manuskripte von Marx nichts mit Statistik zu tun. Es handelte sich vielmehr um eine Auseinandersetzung mit der Differentialrechnung und den Möglichkeiten mit ihrer Hilfe ökonomische Krisen zu beschreiben, vgl. Vogt und Scholz (2018).
} 
Einrichtungen, über das Ausmaß an „Überbelegung“ ... und die Anzahl von Personen (unterschiedlichen Geschlechts) pro Bett" (Schnell et al. 2008, S. 22). Auch wenn diese soziale Bewegung häufig in ihren Annahmen irrte, so hat sie doch erreicht, dass die Regierung auf soziale Probleme aufmerksam wurde, Untersuchungen veranlasste und Untersuchungskommissionen und sozialpolitische Enqueten einrichtete: „Ein Teil des Materials dieser Enqueten wurde von Friedrich Engels in die „Lage der arbeitenden Klasse in England“ (1845) und von Karl Marx im „Kapital“ (1867) innerhalb eines neuen theoretischen Bezugsrahmens ausgewertet und interpretiert" (Schnell et al. 2008, S. 23). Gumbel hatte auch seine Leserschaft aufgerufen, eigenmächtig Zeitungen auszuwerten und Daten über ungerechte Gerichtsverfahren zu veröffentlichen, allerdings ohne Erfolg.

Im August 1930 wurde Gumbel schließlich der Titel eines „Außerordentlichen Professors" verliehen. Die Entscheidung des Kultusministeriums erfolgte gegen die Fakultät. Gegen diese Ernennung machten der Nationalsozialistische Deutsche Studentenbund sowie die Burschenschaften mobil. Überall wurden Gumbels Auftritte mit Tumulten und Schlägereien gestört. So fand sich auf dem Schwarzen Brett des Heidelberger Instituts für Sozial- und Staatswissenschaften ein Flugblatt eines „Aktionsausschuß nationale Studenten“, in dem es unter anderem hieß: „Also ferngeblieben, deutsche Studenten, von den Vorlesungen jüdischer Dozenten. - Das Volk Israel soll sich seine eigene Universität aufmachen, in Jerusalem oder in Frankfurt. Dagegen ist nichts einzuwenden. - Dort kann Herr Gumbel seine Statistik, eine Tätigkeit, die gar nicht unter wissenschaftliches Arbeiten fällt, ausüben, dort kann er auf Gimbelfang ${ }^{5}$ gehen ..." (Jansen 1981, S. 38). Bemerkenswert ist an dieser Stelle, dass hier der Statistik per se der Rang einer Wissenschaft abgesprochen wurde. Diese Brandmarkung ganzer Wissenschaftsdisziplinen führte unmittelbar nach Beginn der NS-Herrschaft zu deren systematischer Abwicklung in Universitäten und Bibliotheken ${ }^{6}$.

Diese Tumulte wurden in Umkehrung der Täterschaft in der Presse als „GumbelKrawalle" ${ }^{\text {"7 }}$ bezeichnet. Höhepunkt der Kampagne war in Heidelberg eine Massenveranstaltung von 3000 Personen, um die „Entfernung Gumbels von Heidelberg“ endgültig durchzusetzen. Aber auch der Verband der deutschen Hochschullehrer, deren offizielle Standesvertretung, begrüßte ,,aufs wärmste“ jede Äußerung, ,in der die Deutsche Studentenschaft ihre vaterländische Gesinnung bekundet" und zeigte „Verständnis“ für die Krawalle. Es gab aber auch öffentliche Unterstützung für Gumbel. Im April 1931 fand auf Anregung der Liga für Menschenrechte in Berlin eine Diskussionsveranstaltung zum „Fall Gumbel“ und der Heidelberger Geschehnisse mit eintausend Besuchern statt. Eine Protesterklärung wurde allerdings nur

\footnotetext{
${ }^{5}$ Gimbelfang (eigentlich „Gimpelfang“) steht hier für Dummenfang.

${ }^{6}$ Als Ersatz wurde eine rassistisch orientierte „Deutsche Mathematik“ propagiert, siehe hierzu den Beitrag von Vogt https://link.springer.com/content/pdf/10.1007.pdf in Bergmann und Epple (2009), S. $193 \mathrm{ff}$.

7 Eine ausführliche Darstellung der Heidelberger Ereignisse findet sich bei Jansen (1981), der das Archiv der Universität unter Einbezug von Flugblättern und Presseberichten zum „Fall Gumbel“ ausgewertet hat. Diese Dokumentation wurde 2012 von Christine Dörfler in einer Internet-Fassung leicht zugänglich gemacht: http://arciv.ub.uni-heidelberg.de/volltextserver/13154/1/jansen.pdf. Siehe auch Brenner (2001) sowie Jansen (1991).
} 
von 80 Hochschullehrern - von damals insgesamt 5000 Professoren in Deutschland - unterzeichnet.

Schließlich kam es 1932 zum inszenierten Eklat. Auf einer nicht-öffentlichen Sitzung der Sozialistischen Studentenschaft zum Thema „Krieg und Arbeiterbewegung“" schrieben drei Mitglieder des Nationalsozialistischen Studentenbundes mit. Gumbel erinnerte in seinem Vortrag an den Hungerwinter 1916/17, in dem sich die Bevölkerung mit Kohlrüben durchschlagen musste: „Für mich ist das Denkmal des Kriegs nicht eine leichtbekleidete Jungfrau mit Siegespalme in der Hand, sondern die Schrecken und das Leid des Kriegs werden viel besser durch eine Kohlrübe verkörpert“ (Jansen 1981, S. 65). Die Zeitung „Der Heidelberger Student“ verkürzte diese Ausführungen zu: „Das Kriegerdenkmal des deutschen Soldaten ist für mich nicht eine leichtbekleidete Jungfrau mit der Siegespalme in der Hand, sondern eine einzige Kohlrübe." Gumbel selber bestritt den Wortlaut. Es half ihm allerdings nichts. Seine eigene Fakultät leitete ein Disziplinarverfahren gegen ihn ein, in dessen Ausgang Gumbel im August 1932 die Lehrbefugnis wegen „Unwürdigkeit““ entzogen wurde.

Ironischerweise rettete dieser Rausschmiss Gumbel das Leben. Er übernahm noch im Herbst desselben Jahres einen Lehrauftrag als Gastprofessor am Institut Poincarè in Paris, so dass er bei Machtantritt der Nationalsozialisten nicht mehr in Deutschland war. Er stand als einer von 33 Personen und als einziger Wissenschaftler auf der ersten von mehr als 300 Ausbürgerungslisten des Innenministeriums. Mit der Ausbürgerung war die Ausraubung verbunden, die als Beschlagnahme des Vermögens zugunsten des Deutschen Reichs kaschiert wurde ${ }^{8}$. Bereits im März 1933, also schon kurz nach Hitlers Machtergreifung, wurde Gumbels Wohnung in Deutschland aufgebrochen und geplündert. Seine Bücher wurden verbrannt. Gumbel arbeitete während seines französischen Exils zunächst in Paris und ab 1934 am Institut für Versicherungswirtschaft der Universität Lyon, siehe Fernández und Scherer (2016). Dort wurde 2016 zu seinem 125. Geburtstag ein Hörsaal, das „Amphi Gumbel“, nach ihm benannt.

1940 gelang ihm nur knapp vor der heranrückenden deutschen Armee die Flucht über Spanien nach Lissabon. Gumbel beschrieb seine abenteuliche Flucht in einem Buch (1941, dt. in Jansen 1991). Er kam mit einem Emergency Visum des USamerikanischen Hilfskommittees und dank des Affidavit (hier als Bürgschaft zu verstehen) der New York School for Social Research in die USA, siehe Gumbel (1959). Einige Jahre später, 1946, wurde er US-Bürger.

Gumbel lehrte zunächst in New York an der New School for Social Research. Sie entwickelte sich zu einer „University in Exile“ für politisch Verfolgte des nationalsozialistischen Regimes und setzte sich für geflüchtete europäische Intellektuelle ein, wie z.B. den Anthropologen Claude Lèvi-Strauss. Später, jedoch erst nach 1945, lehrte dort die Politikwissenschaftlerin und Philosophin Hannah Arendt. Dort bekam Gumbel zunächst einen Lehrauftrag für mathematische Statistik, denn Festanstellungen gab es für Emigranten faktisch nicht. Im Anschluss finanzierte ihn die Rockefeller Foundation bis 1944 ein Forschungsvorhaben zu wahrscheinlichkeitstheoretisch begründeten Vorhersagen von Hochwassern im Mississippi-Flusssystem.

\footnotetext{
${ }^{8}$ Gumbel war stolz, auf der ersten Liste gewesen zu sein. Quelle: Gumbel (1936), zitiert in: Vogt (1991).
} 
Gumbel setzte hier seine Forschungen zu Extremwerten, d.h. maximalen oder minimalen Werten in beobachteten Daten, die er bereits in Lyon begonnen hatte, fort. Zwischen 1946 und 1952 lehrte er an verschiedenen New Yorker Colleges. 1953 erhielt Gumbel eine Anstellung, aber gerade einmal als „Adjunct Professor“, also vergleichbar mit einem „Assistant Professor“, non-tenure-track, an der Columbia University im Department of Engineering in New York. 1958 erschien Gumbels noch heute relevantes wissenschaftliches Hauptwerk „The Statistics of Extremes“, das die Verteilung von Maxima und Minima behandelt. Nicht nur in den USA wurden diese Arbeiten zum Beispiel für die Berechung von Deichhöhen grundlegend, sondern auch in Europa: So, zum Beispiel, lieferten diese Arbeiten nach der verheerenden Sturmflut von 1953, die große Teile des Rhein-Schelde-Deltas unter Wasser setzte, die methodischen Grundlagen für die Neuberechnung von Deichhöhen. In seinen letzten Lebensjahren erforschte Gumbel verschiedene bivariate Wahrscheinlichkeitsverteilungen, so dass es auch eine nach Gumbel benannte Copula gibt, vgl https://de.wikipedia.org/wiki/Copula_(Mathematik). Damit wurde Gumbel nicht nur im Ingenieur-Bereich, sondern auch im Versicherungs- und im Finance-Bereich eine wichtige Größe, vgl. Fernández und Scherer (2016).

Gumbel hat versucht, dass seine Entlassung wegen „Unwürdigkeit“ zurück-genommen wird. Doch an der Heidelberger Universität bedeutete man ihm, dass man seine Wiedereinsetzung als Professor zu verhindern wisse. Darüber hinaus wurden auch Gumbels Forderungen auf Wiedergutmachungszahlungen zunächst abgelehnt. Denn da er bereits 1932 entlassen worden war, so die Begründung, könne er keine Zahlungen nach dem „Gesetz zur Wiedergutmachung nationalsozialistischen Unrechts" für sich in Anspruch nehmen. Erst durch die Hilfe Robert Kempners, des stellvertretenden US-Chefanklägers bei den Nürnberger Kriegsverbrecherprozessen, gelang es Gumbel 1956, Pensionsansprüche vom Land Baden-Württemberg zu erstreiten ${ }^{9}$, vgl Kempner (1983).

Gumbel lehrte einige Jahre als Gastprofessor an einigen deutschen Universitäten. Von 1953 bis 1956 lehrte er jeweils im Sommersemester am Mathematischen Institut der Freien Universität Berlin, Vogt (2021). Sein politisches Engagement blieb ungebrochen: So setzte er sich mit den politischen Verhältnissen in Deutschland Anfang der 50er Jahre kritisch auseinander, wo er gegen die Wiederbewaffnung protestierte. Noch kurz vor seinem Tod engagierte er sich bei Protesten gegen den Vietnam-Krieg. Emil Julius Gumbel verstarb am 10. September 1966 in New York an Lungenkrebs im Alter von 75 Jahren.

Trotz seiner enormen Populartät in der Weimarer Republik erschien in Deutschland kein einziger Nachruf ${ }^{10}$ ! Anläßlich seines 100. Geburtstags gab es an der Universität Heidelberg eine Gedenkveranstaltung zu Ehren Gumbels. Der damalige Rektor Peter Ulmer erklärte: „Dass die inkriminierten, mit der Forschungs- und Lehrtätigkeit von Gumbel in keinem unmittelbaren Zusammenhang stehenden politischen Akti-

\footnotetext{
9 Wie in ähnlichen Fällen war die Zahlung der Pensionsansprüche an diskriminierende Auflagen gebunden. Die Zahlungen gingen auf ein Sperrkonto, von dem nur Zahlungen in Deutschland beglichen werden konnten. Die davon erworbenen Güter mußten dann in die USA ausgeführt werden, vgl. Vogt (2021).

${ }^{10}$ Lediglich sein alter Kampf- und Exilgefährte Willi Eichler veröffentlichte einen Nachruf, siehe Eichler (1966).
} 
vitäten aus heutiger Sicht keinen hinreichenden Grund zu einer derartigen Entscheidung geben würden, steht für mich vor dem Hintergrund der verfassungsrechtlich geschützten allgemeinen Meinungsäußerungsfreiheit außer Zweifel.“ Diese strikte Trennung von universitärer Lehre und politischer Überzeugung bestätigt auch einer seiner früheren Studenten und späterer Professor am Engeneering Department, Tuncel Yegulalp, in einem Interview über Gumbels letzte Vorlesung. (siehe Fernández und Scherer 2018)

Einer der Nachfolger Gumbels auf dem Lehrstuhl für Statistik, Hartmut Kogelschatz, betonte ,welchen großen Verlust die deutsche Wissenschaft durch den Entzug seiner Venia Legendi an der Universität Heidelberg im Jahre 1932 erlitt“.

\section{Extremwerte und die Gumbel Verteilung}

Gumbels Publikationen drehten sich zwischen 1933 bis 1940 in Frankreich um bevölkerungsstatistische Probleme und in zunehmenden Maße, vor allem nach 1940 in den USA, um das Verhalten von Extremwerten. Worum es dabei geht und welche praktische Bedeutung die Theorie der Extremwerte haben kann, sagt Gumbel in einem Interview: „Meine eigenen Arbeiten sind ein sehr enger Bereich, nämlich die Statistik der größten Werte [auch Extremwerttheorie genannt]. Das bedeutet, dass ich mich im Gegensatz zu den Hauptarbeiten der mathematischen Statistik nicht mit Mittelwerten und häufigen Ereignissen beschäftige. Praktische Anwendungen hat dieses Gebiet gefunden in der Hydrologie, die sich mit den Durchflussmengen durch einen Fluss beschäftigt, und ich glaube, dass meine Theorie der Überschwemmungen und der Dürre (...) bestimmte praktische Bedeutungen bekommen hat. Andere Anwendungen sind die Dauerfestigkeit von Materialien unter wiederholten Beanspruchungen. Das kann für die Sicherheit von Konstruktionen von Bedeutung sein“ (Gumbel 1959).

Die Verteilung des größten Wertes $X_{\max }$ in einer Stichprobe vom Umfang $n$ lässt sich leicht finden: $X_{\max }$ ist genau dann kleiner oder gleich einem vorgegebenen Wert $x$, wenn alle $n$ Werte $X_{i} ; i=1, \ldots, n$ der Stichprobe kleiner oder gleich $x$ sind. Unter der Annahme, dass jeder der Werte $X_{i}$ derselben Verteilung mit der Verteilungsfunktion $F(\cdot)$ folgt, ist diese Wahrscheinlichkeit für $X_{i} \leq x$ gleich $F(x)$. Und unter der weiteren Annahme, dass die $X_{i}$ unabhängig sind, ist die Wahrscheinlichkeit dafür, dass $X_{\max } \leq X$ ist, gleich

$$
\begin{aligned}
G(x)=P\left(X_{\max } \leq x\right) & =P\left(X_{1} \leq x \text { und } X_{2} \leq x \text { und } \ldots \text { und } X_{n} \leq x\right) \\
& =P\left(X_{1} \leq x\right) P\left(X_{2} \leq x\right) \ldots P\left(X_{n} \leq x\right)=F^{n}(x)
\end{aligned}
$$

Von der Überlegung ausgehend, dass der kleinste Wert in einer Stichprobe genau dann größer oder gleich einem gegebenen Wert $x$ ist, wenn alle Werte der Stichprobe größer oder gleich $x$ sind, gelangt man zur Verteilungsfunktion des kleinsten Wertes $X_{\min }$ einer Stichprobe: $G(x)=1-(1-F(x))^{n}$. Die zugehörigen Wahrscheinlichkeitsdichtefunktionen ergeben sich zu $g(x)=n F^{n-1}(x) f(x)$ für $X_{\max }$ und $g(x)=n(1-F(x))^{n-1} f(x)$ für $X_{\min }$. Abb. 3 zeigt Wahrscheinlichkeitsdichtefunktionen von $X_{\max }$, falls die Verteilung von $X$ eine Normalverteilung ist; der Ein- 


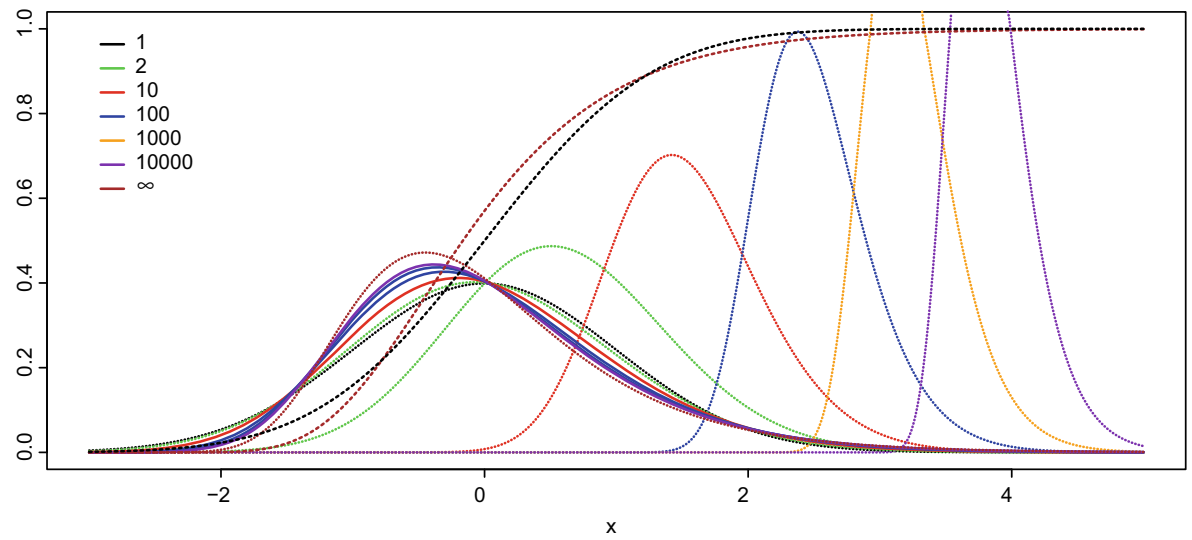

Abb. 3 Die Wahrscheinlichkeitsdichtefunktionen der Normalverteilung, der Verteilungen von $X_{\max }$ für $n=2,10,100,10000,10000$ (punktierte Linien) und die der jeweils auf $\mu=0$ und $\sigma=1$ standardisierten Verteilungen (durchgezogene Linien) sowie der Typ I-Asymptote als Grenzverteilung. Die Verteilungsfunktionen für $n=1$ und $n=\infty$ sind gestrichelt eingezeichnet

fachheit halber wurde die standardisierte Normalverteilung (Erwartungswert $\mu=0$ und Standardabweichung $\sigma=1)$ gewählt. Wie zu erwarten, sind die größten Werte tendenziell größer als die Einzelwerte, d.h. die Wahrscheinlichkeitsdichtefunktionen von $X_{\max }$ verschieben sich mit wachsendem $n$ nach rechts; außerdem werden sie schmaler, d.h. $X_{\max }$ streut mit wachsendem $n$ weniger, hat also eine kleinere Standardabweichung. Standardisiert man die Verteilungen von $X_{\max }$, d.h. transformiert man $X_{\max }$ zu $Y_{\max }=\left(X_{\max }-\mu_{X_{\max }}\right) / \sigma_{X_{\max }}$, dann kann man sie für unterschiedliche $n$ direkt vergleichen und erkennt, dass die Verteilungen keine Normalverteilungen sind - auch dann nicht, wenn die Verteilung von $X$ eine Normalverteilung ist. Mit wachsendem $n$ streben die Verteilungen einer Grenzverteilung zu, die gestrichelt in Abb. 3 eingezeichnet ist. Die Form der Grenzverteilung von $X_{\max }$ hängt von der Form der Verteilung von $X$ ab. Das hat etwa ab 1920 ein weites Forschungsfeld geöffnet, in dem fast alle großen Statistiker des 20. Jahrhunderts tätig waren. Wohl der erste war 1922 Ladislaus von Bortkiewicz (von Bortkiewicz 1922), der sich mit Extremwerten bei Normalverteilung befasste, und vermutlich hat er auch Gumbel angeregt, sich damit zu befassen (Haerdle und Vogt 2015). Leonard H. C. Tippett (Tippett 1925) berechnete 1925 numerische Werte der Wahrscheinlichkeiten dieser Verteilungen, Richard von Mises (von von Mises 1923) befasste sich 1923 mit asymptotischen Extremwertverteilungen und Maurice René Fréchet (Fréchet 1927) leitete 1927 die asymptotische Extremwertverteilung her, die später Typ IIExtremwertverteilung oder Fréchet-Verteilung genannt wurde. Ronald A. Fisher und Leonard H. Tippett (Fisher und Tippett 1928) leiteten 1928 neben der von Fréchet gefundenen Typ II-Asymptote noch zwei weitere Asymptoten her und zeigten, dass es keine weiteren geben kann, mit folgendem Argument: Angenommen, es liegen $m$ Stichproben vom Umfang $n$ und deren größte Werte vor. Der größte Wert dieser $n$ größten Werte ist auch der größte Wert, der aus den $m$ Stichproben gebildeten Stichprobe vom Umfang $n \cdot m$. Die asymptotische Verteilung einer Stichprobe vom Umfang $m \cdot n$ muss also dieselbe sein wie die des größten Wertes einer Stichprobe 
vom Umfang $n$ - bis auf eine lineare Transformation von $X_{\max }$. Für die Verteilungsfunktion von $X_{\max }$ muss also gelten:

$$
G^{n}(x)=G\left(a_{n} x+b_{n}\right) .
$$

Jede asymptotische Verteilungsfunktion $G(x)$ des größten Wertes muss diese Funktionalgleichung erfüllen, und es lässt sich leicht zeigen, dass es nur 3 verschiedene Verteilungstypen $G(x)$ gibt. Sie werden als Asymptoten vom Typ I, II und III bezeichnet. Inzwischen heißen sie Gumbel-Verteilung, Fréchet-Verteilung und Weibull-Verteilung.

Die interessante Frage, wieviele verschiedene asymptotische Extremwertverteilungen es gibt und welche Ausgangsverteilungen $\mathrm{zu}$ welcher Asymptote führen, wurde insbesondere von Boris V. Gnedenko (Gnedenko 1943) untersucht. Der Satz, dass die asymptotische Extremwertverteilung, wenn sie überhaupt existiert, eine der drei Asymptoten ist, heißt inzwischen Satz von Fisher-Tippett-Gnedenko. Unter den Asymptoten haben zwei eine große praktische Bedeutung. Eine ist die Typ I-Asymptote des Maximums, die Gumbel-Verteilung, eine andere die Typ III-Asymptote des Minimums, für die sich der Name Weibull-Verteilung durchgesetzt hat.

Mit der Gumbel-Verteilung lassen sich Extremwertdaten häufig gut modellieren, möglicherweise deshalb, weil sie sich u. a. bei der Normalverteilung als Ausgangsverteilung ergibt. Gumbel hat sich intensiv mit Schätz- und Testverfahren bei dieser Verteilung befasst und diese in vielen verschiedenen Gebieten angewendet, wie etwa bei radioaktiver Strahlung (Gumbel 1936), extremen Tagestemperaturen (Gumbel 1940), extremen Wassermengen von Flüssen (Gumbel 1941), extremen Werten in der Meteorologie (Gumbel 1942), in der Hydrologie (Gumbel 1943) und in der Luftfahrt (Gumbel und Carlson 1954), bei Ermüdungserscheinungen von Metallen (Gumbel 1955) und bei der Untersuchung von Dauerschwingfestigkeiten (Gumbel 1956). Das ist nur ein kleiner Teil der Arbeiten Gumbels auf diesem Gebiet. Da Gumbel seit 1940 immer mit Naturwissenschaftlern und Ingenieuren zusammen gearbeitet hat, waren die von ihm entwickelten Methoden direkt in der Praxis anwendbar. Er hat insbesondere auf graphische Analysemethoden Wert gelegt, vor allem deshalb, weil damals wegen des Fehlens von Rechnerkapazitäten viele numerische Methoden nicht praktikabel waren. Von ihm stammt das Wahrscheinlichkeitsnetz zur Auswertung von Gumbel-verteilten Daten (Gumbel-Netz). Es beruht darauf, dass die GumbelVerteilung zur Lage- und Skalenfamilie gehört, d.h. ihre Verteilungsfunktion,

$$
G(x)=\exp (-\exp (-(x-\alpha) / \beta)), \quad x \in \mathbb{R}
$$

mit dem Lokationsparameter $\alpha \in \mathbb{R}$ und dem Skalenparameter $\beta 0$, lässt sich durch lineare Transformation der Zufallsvariablen $X \mathrm{zu} Y=(X-\alpha) / \beta$ in die parameterfreie ,reduzierte“ Verteilungsfunktion $G(y)=\exp (-\exp (-y))$ überführen. Logarithmiert man zweimal, dann erhält man die lineare Gleichung $-\ln (-\ln (G))=$ $y=(x-\alpha) / \beta$ zwischen $y=-\ln (-\ln (G))$ und $x$. In einem Netz mit der Ordinatenteilung $-\ln (-\ln (G))$ f"ur $G$ und der Abszissenteilung $x$ ist daher die Verteilungsfunktion jeder Gumbel-Verteilung eine Gerade. Die empirische Verteilungsfunktion einer Stichprobe lässt sich, falls die Daten aus einer Gumbel-Verteilung stammen, 


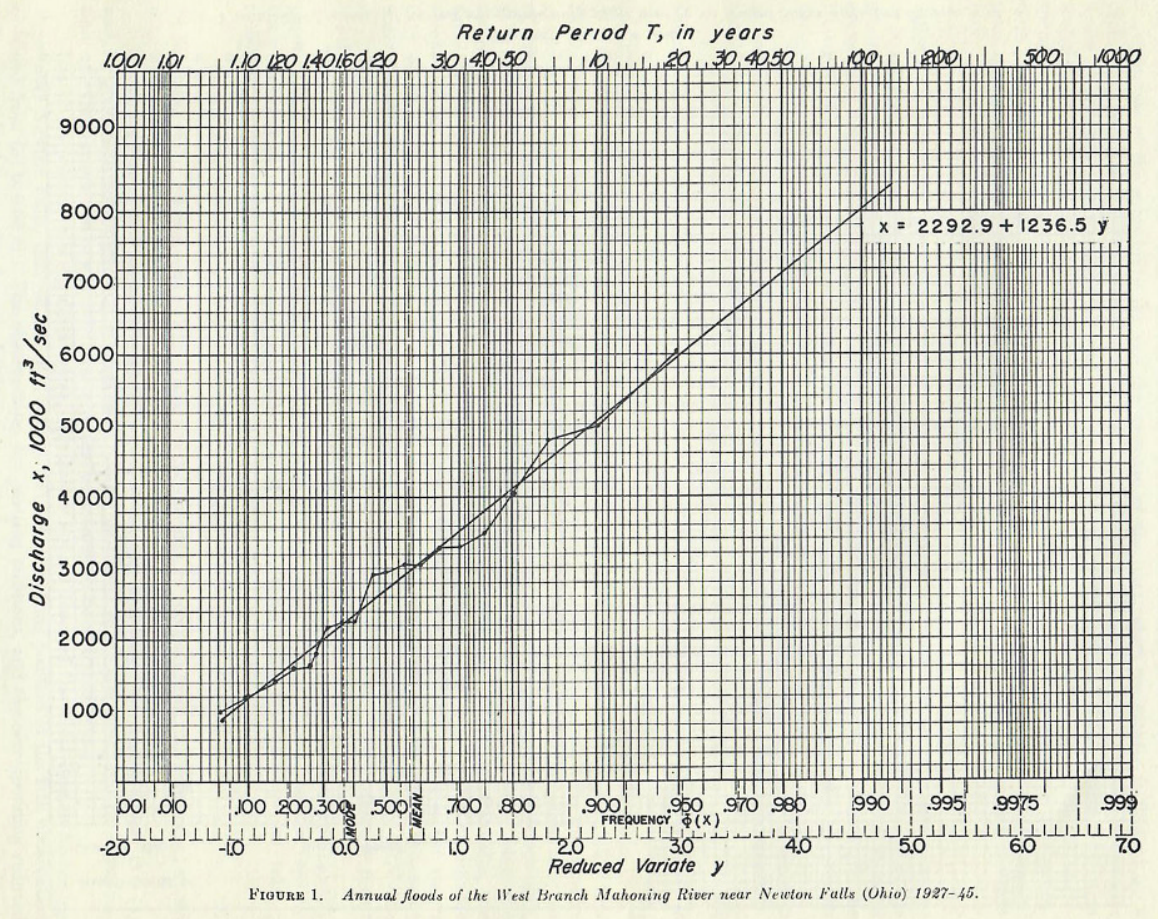

Abb. 4 Die jährlichen Höchstdurchflussmengen des West Branch Mahoning Flusses in der Nähe von Newton Falls (Ohio, USA) 1927 bis 1945 im Gumbel-Netz

im Gumbel-Netz zwanglos durch eine Gerade ausgleichen, an der sich Schätzwerte für die beiden Parameter $\alpha$ und $\beta$ bestimmen lassen. Abb. 4 zeigt die Jahreshöchstdurchfluss-mengen (in $1000 \mathrm{ft} 3 / \mathrm{sec}$ ) eines Flusses in den 19 Jahren 1927 bis 1945 im Gumbel-Netz (Abszisse und Ordinate sind vertauscht; anstelle der empirischen Verteilungsfunktion ist eine leicht davon abweichende, für die Auswertung besser geeignete Variante der Verteilungsfunktion eingezeichnet) und die Schätzwerte für $\alpha$ und $\beta$ wurden graphisch bestimmt. Beispielsweise liest man ab, dass eine Höchstdurchflussmenge von 8000 , d.h. $8 \mathrm{ft} 3 / \mathrm{sec}$ mit der (geschätzten) Wahrscheinlichkeit 0,99 unter-, also mit der (geschätzten) Wahrscheinlichkeit 0,01 überschritten wird. Wie Statistiker ganz allgemein hat wohl auch Gumbel feststellen müssen, dass Wahrscheinlickeiten von Laien oft falsch interpretiert werden und hat deshalb eine neue Kenngröße, die Wiederkehrperiode (return period), eingeführt: Wenn beispielsweise die Wahrscheinlichkeit des Erreichens oder Überschreitens einer Höchstdurchflussmenge gleich 0,01 ist, dann erwartet man dieses Ereignis in einem von 100 Jahren, oder anders gesagt: man erwartet es in Abständen von 100 Jahren, wie in Abb. 4 an der Skala am oberen Rand ablesbar ist. Die Wiederkehrperiode, die der Reziprokwert der Überschreitungswahrscheinlichkeit ist, ist anschaulicher interpretierbar, aber man sollte natürlich im Kopf haben, dass ein Ereignis, das man nach 100 Beobachtungen erwartet, durchaus auch als nächstes beobachtet werden kann, oder auch erst nach mehreren 100 Beobachtungen. 
Abb. 5 Abgangslinien für einige wirtschaftliche Gesamtheiten: $G$ englische Aktiengesellschaften um 1900; F Einzelhandelsgeschäfte des Staates Illinois, USA, um 1932; K Kolonialwarengeschäfte in Buffalo um 1925; J Beschäftigungsverhältnisse in der italienischen Textilindustrie um 1925; B Beschäftigungsverhältnisse in der britischen Industrie um 1949 (Stange 1955, S. 141)

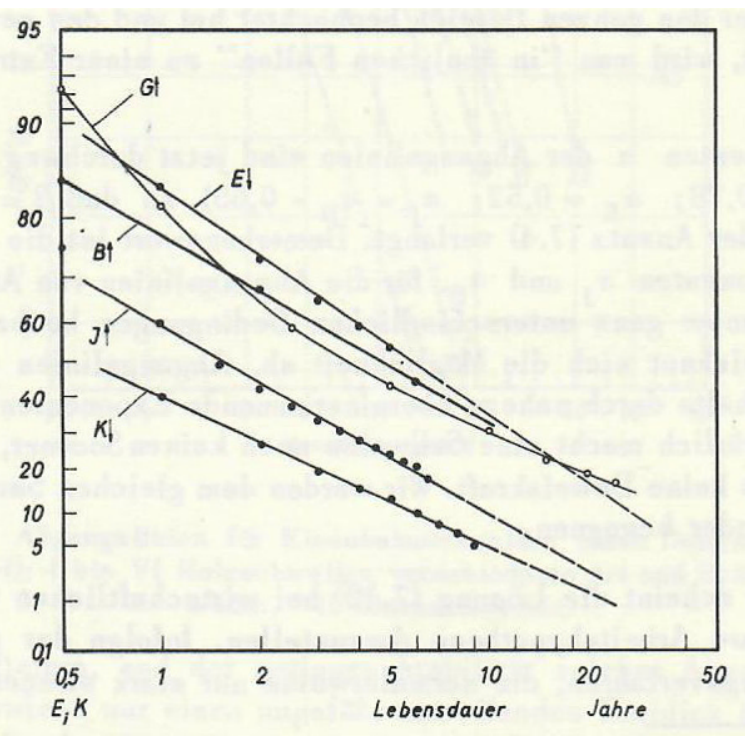

Die Weibull-Verteilung, die Typ III-Asymptote des Minimums, läßt sich durch logarithmische Transformation der Ausgangsvariablen $x \mathrm{zu} y=\ln x$ in die Gumbel-Verteilung transformieren, so dass sich bei Vorliegen von Weibull-Verteilung die Logarithmen als Gumbel-verteilt auswerten lassen, insbesondere auch graphisch einfach dadurch, dass die $x$-Achse im Gumbel-Netz durch eine Achse ln $x$ ergänzt oder ersetzt wird (Weibull-Netz). Mit der Weibull-Verteilung, mit der sich Gumbel auch befasst hat, lassen sich insbesondere Lebensdauerdaten (von technischen Komponenten und Aggregaten) und Daten aus Festigkeitsuntersuchungen gut modellieren. Das liegt nahe, denn man kann sich den beobachteten Wert der Lebensdauer oder der Festigkeit einer Betrachtungseinheit als Minimum von (nicht beobachteten) Lebensdauern bzw. Festigkeiten vorstellen, die verschiedene Ursachen haben (eine Kette ist so stark wie ihr schwächstes Glied). Der schwedische Ingenieur und Technologieprofessor Waloddi Weibull fand 1939 rein empirisch heraus (Weibull 1939a,b), wie auch Paul O. Rosin, Erich Rammler und Karl Sperling (Rosin und Rammler 1933), dass sich Sieblinien von Schüttgütern mit diesem Verteilungmodell sehr oft gut modellieren lassen. Schüttgüter wie zum Beispiel Kohle, Kalk oder Getreide bestehen aus Teilchen („Körnern“) unterschiedlicher Durchmesser bzw. Gewichte. Schüttet man eine Probe durch aufeinander gestellte Siebe, deren Maschenweiten von oben nach unten kleiner werden und wiegt die Rückstände aus, dann hat man direkt eine empirische Verteilungsfunktion des Siebrückstands, die sich im Weibullbzw. Gumbel-Netz direkt auswerten lässt. Bei der Analyse von Sieblinien heißt das Weibull-Netz „Körnungsnetz“ oder „RRSB-Netz“ (nach Rosin, Rammler, Sperling, Bennett; Karl Sperling und John G. Bennett haben das von Rosin und Rammler entworfene Netz in eine praktischere Form gebracht) und hat eine so große Bedeu- 
Abb. 6 Abgangslinien für Eisenbahnschwellen; I bis VI

Holzschwellen verschiedener Art und Behandlungsweise, VII Eisenschwellen (Stange 1955, S. 143)

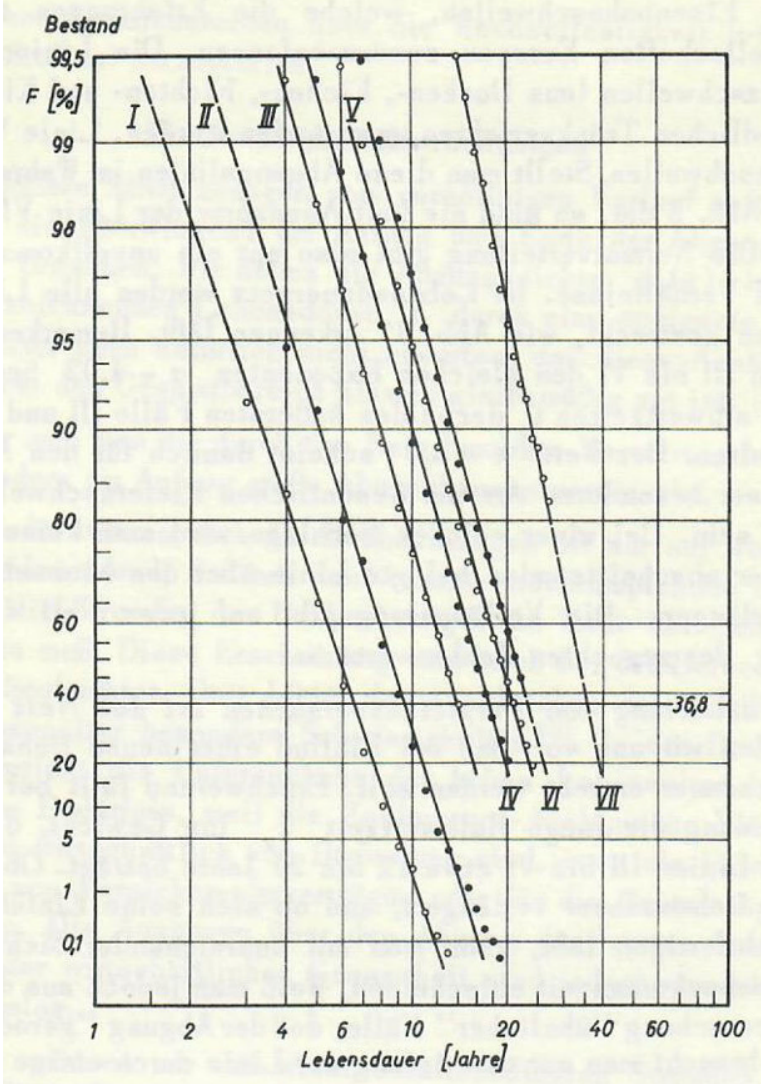

tung, dass es beispielsweise in der Norm DIN 66145 ${ }^{11}$ zur Verfügung gestellt wird. Kurt Stange (Stange 1955) präsentiert 1955 Beispiele von Daten aus unterschiedlichen Gebieten, die sich gut mit der Weibull-Verteilung, deren Logarithmen also gut mit der Gumbel-Verteilung ausgewertet werden können. In seinem Netz, das er Lebensdauernetz genannt hat, wird nicht die empirische Verteilungsfunktion $G(x)$, sondern die Abgangslinie $1-G(x)$ eingetragen, also die Bestandsverringerungen in einer Stichprobe von Beginn (Bestand ,1“) bis zu großen $x$, bei denen der Bestand der noch ,lebenden“ Einheiten wegen der Ausfälle gegen Null geht. Abb. 5 zeigt Abgangslinien von wirtschaftlichen Gesamtheiten, Abb. 6 Abgangslinien für Eisenbahnschwellen.

Gumbel hat, wie er selbst sagt, seit 1949 an seiner Monographie über Extremwertverteilungen gearbeitet. Das 1958 erschienene Buch „Statistics of Extremes“ (Gumbel 1958, Reprint 2013) war für viele Jahre die einzige zusammenfassende Darstellung der Extremwertheorie und ist auch heute noch wegen der vielen darin präsentierten statistischen Analysemethoden ein Buch, das man gern benutzt.

11 DIN 66145 (1976) Darstellung von Korn-(Teilchen-)größenverteilungen; RRSB-Netz. 
Gumbel schreibt in der Einleitung: ,This book is written in the hope, contrary to expectation, that humanity may profit by even a small contribution to the progress of science."

Dieser Satz zeigt Gumbels Bescheidenheit, die man auch bei seinem Besuch 1965 in Aachen immer wieder beobachten konnte. Gumbel war damals 74 Jahre alt, wirkte aber viel jünger und war an allem interessiert. Ich, Peter-Theodor Wilrich, werde nie vergessen, wie er in dieser Besuchswoche einmal vorsichtig an die Tür meines Zimmers klopfte und fragte, ob er mich für eine kurze Zeit stören dürfe. Er wolle gern erfahren, womit ich mich beschäftige. Wir haben dann etwa zwei Stunden über meine Arbeit an der Dissertation zu einem Warteschlangenthema gesprochen. Am Schluss des Gesprächs bot er mir an, mir mit Rat zur Verfügung zu stehen, wenn ich Hilfe brauche. Ein solches Interesse an meiner Arbeit und die damit verbundene Hilfsbereitschaft ist mir weder vor diesem Gespräch noch anschließend jemals wieder begegnet.

Gumbel war ein bedeutender er Statistiker der ersten Hälfte des 20. Jahrhunderts. Die von ihm entwickelten Methoden der statistischen Analyse von Extremwerten werden ihre Bedeutung behalten. Keinen Erfolg hatte er mit seinen Publikationen und Reden im Kampf gegen die Nationalsozialisten und für Freiheit und Gerechtigkeit. Er war leider nur einer von sehr wenigen, die die Gefahr des Nationalsozialismus sehr frühzeitig erkannt haben. In seinem Einstehen für eine freiheitliche Demokratie sollte er uns ein Vorbild sein.

Danksagung Unser herzlicher Dank gilt Anette Vogt vom Max-Planck-Institut für Wissenschaftsgeschichte. Sie hat uns als Nicht-Historikern ihren reichen Fundus an Quellen über Gumbel eröffnet und geduldig unsere erste Manuskriptfassung korrigiert und ergänzt. In einem persönlichen Gespräch im Harnack-Haus in Berlin-Dahlem konnten wir unser Bild von Gumbel vertiefen. Dabei verstärkte sich der Eindruck, wie ahnungslos wir als Statistiker gegenüber dem ,politischen Gumbel“ waren. Umso mehr sind wir für diesen Nachhilfeunterricht dankbar. Wir hoffen auf eine ähnliche Reaktion bei unseren Lesern.

Funding Open Access funding enabled and organized by Projekt DEAL.

Open Access Dieser Artikel wird unter der Creative Commons Namensnennung 4.0 International Lizenz veröffentlicht, welche die Nutzung, Vervielfältigung, Bearbeitung, Verbreitung und Wiedergabe in jeglichem Medium und Format erlaubt, sofern Sie den/die ursprünglichen Autor(en) und die Quelle ordnungsgemäß nennen, einen Link zur Creative Commons Lizenz beifügen und angeben, ob Änderungen vorgenommen wurden.

Die in diesem Artikel enthaltenen Bilder und sonstiges Drittmaterial unterliegen ebenfalls der genannten Creative Commons Lizenz, sofern sich aus der Abbildungslegende nichts anderes ergibt. Sofern das betreffende Material nicht unter der genannten Creative Commons Lizenz steht und die betreffende Handlung nicht nach gesetzlichen Vorschriften erlaubt ist, ist für die oben aufgeführten Weiterverwendungen des Materials die Einwilligung des jeweiligen Rechteinhabers einzuholen.

Weitere Details zur Lizenz entnehmen Sie bitte der Lizenzinformation auf http://creativecommons.org/ licenses/by/4.0/deed.de.

\section{Literatur}

Benz W (1983) Emil J. Gumbel. Die Karriere eines deutschen Pazifisten. In: Walberer U (Hrsg) 10. Mai 1933. Bücherverbrennung in Deutschland und die Folgen. , Frankfurt am Main, S 160-198 
Bergmann B, Epple M (Hrsg) (2009) Jüdische Mathematiker in der deutschsprachigen akademischen Kultur. Springer, Heidelberg https://doi.org/10.1007/978-3-540-69252-2

von Bortkiewicz L (1922) Variationsbreite und mittlerer Fehler. Sitzungsber Berl Math Ges 21:3-33

Brenner AD (2001) Emil J. Gumbel. Weimar German Pacifist and Professor. Brill, Leiden

Chlumsky J (2012) Zur Heinz-Grohmann-Vorlesung der Deutschen Statistischen Gesellschaft. AStA Wirtsch Sozialstat Arch 6(1):61-63

Desrosières A (2005) Die Politik der großen Zahlen. Eine Geschichte der statistischen Denkweise. Springer, Berlin

Eichler W (1966) Zum Tode von Emil Gumbel. Geist Tat Monatsschr Recht Freih Kult 21(10):289-292

Fernández L, Scherer M (2016) Emil Julius Gumbel - Festakt zum 125. Geburtstag. In: Der Aktuar 03.2016, S 176-177

Fernández L, Scherer M (2018) Emil Julius Gumbel's last course on the „Statistical theory of extreme values": A conversation with Tuncel M. Yegulalp. Extremes 21:97-113

Fernández L, Scherer M, Vogt A (Hrsg) (2019) Harold Gumbel. Memories. Edition Hentrich Hentrich, Leipzig, Berlin

Fisher RA, Tippett LHC (1928) Limiting forms of the frequency distribution of the largest and smallest member of a sample. Proc Camb Philos Soc 24:180-190

Förster B (2019) Mit Statistik gegen rechte Gewalt. Frankfurter Allgemeine Zeitung. Faz.Net. https:// www.faz.net/aktuell/karriere-hoch-schule/mathematiker-emil-j-gumbel-mit-statistik-gegen-rechtegewalt-16319833-p3.html. Zugegriffen: 21. März 2021

Fréchet MR (1927) Sur la loi de probabilité de l'écart maximum. Ann Soc Polonaise Mathematique 6:93-116

Furth D (2012) Statistiker Emil Gumbel. Rechnen gegen der Terror. Spiegel Geschichte. https://www. spiegel.de/geschichte/statistiker-emil-gumbel-a-947548.html. Zugegriffen: 21. März 2021

Gnedenko BV (1943) Sur la distribution limite du term maximum d'une serié aléatoire. Ann Math Stat 44:423-453

Gumbel EJ (1936) Les inondations et la théorie de la plus grande valeur. Les distances extrèmes entre les émissions radioactives. C R Acad Sci 203:27-29

Gumbel EJ (1919) Vier Jahre Lüge. Flugschriften des Bundes Neues Vaterland, Nr. 5. , Berlin

Gumbel EJ (1921) Zwei Jahre Mord. Neues Vaterland, Berlin

Gumbel EJ (1922) Vier Jahre politischer Mord. Das Wunderhorn, Heidelberg

Gumbel EJ (1940) La durée de retour des témperatures annuelles extrèmes. C R Acad Sci 210:468

Gumbel EJ (1941) The return periods of flood flows. Ann Math Stat 12:163-190

Gumbel EJ (1942) On the frequency distribution of extreme values in meteorological data. Bull Am Meteorol Soc 23:95-105

Gumbel EJ (1943) Statistical analysis in hydrology. Proc Am Soc Civ Eng 69:995

Gumbel EJ (1955) Statistische Theorie der Ermüdungserschei-nungen von Metallen. Mitteilungsbl Math Stat 8:97-130

Gumbel EJ (1956) Programm für die statistische Schätzung der Dauerschwingfestigkeit. Schweiz Arch Angew Wiss Tech 22:375

Gumbel EJ (1958) Statistics of extremes. Columbia University Press, New York

Gumbel EJ (2001) Interview mit Radio Bremen (16.04.1959). In: Vogt A (Hrsg) Emil Julius Gumbel im Interview - Zum 35. Todestag, Bd. Sitzungsberichte der Berliner Mathematischen Gesellschaft, Jg. 1997-2000. Vorstand der BMG, Berlin, S 247-258

Gumbel EJ, Carlson PG (1954) Extreme values in aeronautics. J Aeronaut Sci 21:389-398

Haerdle WK, Vogt AB (2015) Ladislaus von Bortkiewicz - statistician, economist and a European intellectual. Int Stat Rev 83:17-35

Heither D (2016) Ich wusste, was ich tat. Emil Julius Gumbel und der rechte Terror in der Weimarer Republik. PapyRossa, Köln

Heyde CC, Seneta E (Hrsg) (2001) Statisticians of the centuries. Springer, New York, S 406-410

Holl K (1988) Pazifismus in Deutschland. Suhrkamp, Frankfurt am Main

Jansen C (1981) Der „Fall Gumbel“ und die Heidelberger Universität 1924-32. Archiv der Universität Heidelberg. http://archiv.ub.uni-heidelberg.de/volltextserver/13154/1/jansen.pdf. Zugegriffen: 9. Apr. 2020

Jansen C (1991) Emil Julius Gumbel. Porträt eines Zivilisten. Wunderhorn, Heidelberg

Johnson NL, Kotz S (Hrsg) (1997) Leading personalities in statistical sciences: from the seventeenth century to the present. Wiley, New York, S 192-193

Kempner R (1983) Ankläger einer Epoche. Lebenserinnerungen. In Zusammenarbeit mit Jörg Friedrich. Ullstein, Frankfurt/M 
Lahusen B (2012) Das rechte Auge. - Kämpfer gegen die Rechtsterroristen der Weimarer Republik. In: Die Zeit, Nr. 07/2012 (09.02.2012). https://www.zeit.de/2012/07/Gumbel?utm_referrer=https. Zugegriffen: 24. Juni 2021

von Mises R (1923) On the range in a series of observations. Sitzungsber Berl Math Ges 22:3-8

Rosin PO, Rammler E (1933) Laws governing the fineness of powdered coal. J Inst Fuel 7:29-36

Salsburg DS (2001) The lady tasting Tea. How statistics revolutionized science in the twentieth century. In: Chapt. 6: the hundred-year flood. Henry Holt Company, New York, S 53-59

Schnell R, Hill PB, Esser E (2008) Methoden der empirischen Sozialforschung. , München, Wien, S 17-48

Stange K (1955) Zur Ermittlung der Abgangslinie für wirtschaftliche und technische Gesamtheiten. Mitteilungsbl Math Stat 7:113-151

Tippett LHC (1925) On the extreme individuals and the range of samples taken from a normal population. Biometrika 17:364-387

Vogt A (Hrsg) (1991) Emil Julius Gumbel. Auf der Suche nach Wahrheit. Dietz, Berlin

Vogt A (2021) Emil J. Gumbel - Mathematiker, Pazifist und politischer Autor. Sitzungsber Leibniz Sozietät $145: 145-165$

Vogt A, Scholz E (2018) Eintrag „Mathematische Manuskripte“. In: Haug WF et al (Hrsg) Historisch kritisches Wörterbuch des Marxismus, Bd. 9/1. Argument, Hamburg, S 331-341

Weibull W (1939a) A statistical theory of the strength of material. Ingeniörs Vetenskaps Akademiens Handligar Report No. 151. , Stockholm

Weibull W (1939b) The phenomenon of rupture in solids. Ingeniörs Vetenskaps Akademiens Handligar Report No. 153. , Stockholm

Hinweis des Verlags Der Verlag bleibt in Hinblick auf geografische Zuordnungen und Gebietsbezeichnungen in veröffentlichten Karten und Institutsadressen neutral. 